Reprod. Nutr. Dévelop., 1985, 25 (4 B), 787-802.

\title{
Aspects moléculaires de l'adaptation des enzymes pancréatiques et intestinales au régime alimentaire
}

\author{
A. PUIGSERVER, Catherine WICKER, Christine GAUCHER.
}

Centre de Biochimie et de Biologie moléculaire du C.N.R.S., BP 71, 13402 Marseille Cedex 9, France.

Summary : Molecular aspects of dietary adaptation of pancreatic and intestinal hydrolases

The levels of digestive enzymes are known for a long time to be modulated by dietary manipulations as well as genetic and hormonal factors. Adaptation of both types of enzymes, secretory pancreatic hydrolases and intestinal membrane-bound enzymes, to changes in diet has been shown to result from specific variations in the rate of their biosynthesis. As a first step towards the elucidation of the mechanism underlying adaptation of pancreatic enzymes to nutritional substrates we have measured the levels of translatable mRNAs coding for amylase, lipase, serine proteases and procarboxypeptidases. Our results indicate that dietary regulation occurs at a transcriptional level but also involves in some cases changes in mRNA stability. With respect to intestinal enzymes, reliable quantitative data were obtained by activity determination and monodimensional immunoelectrophoresis in response to changes in diet.

\section{Introduction.}

L'existence d'une adaptation du niveau des enzymes digestives aux conditions alimentaires est un avantage physiologique pour l'homme et les animaux supérieurs qui leur permet sans doute d'utiliser plus efficacement les constituants chimiques et l'énergie nécessaires à leur métabolisme. La réponse des systèmes digestifs à l'action d'un nombre croissant d'hormones, ou la mise en évidence d'un contrôle direct de l'expression des gènes de quelques hydrolases dans certains groupes ethniques, démontre clairement l'importance de ce type de régulation.

Depuis les observations historiques de Pavlov, à la fin du siècle dernier, sur la teneur élevée en activités protéolytiques du suc pancréatique chez le chien consommant un repas riche en viande, un très grand nombre de travaux ont abouti à la description précise de phénomènes d'adaptation nutritionnelle. Cependant, les mécanismes par lesquels l'ingestion de quantités extrêmes d'un aliment se traduit par des fluctuations du niveau des hydrolases impliquées dans sa digestion sont encore en grande partie inconnus. II est probable toutefois que la régulation de la biosynthèse des enzymes pancréatiques et intestinales, dont les spécificités d'action sont complémentaires, repose sur des bases moléculaires très compara- 
bles sinon identiques, malgré une complexité différente des tissus correspondants.

Les développements récents des méthodes d'isolement et de traduction des ARN messagers en milieu acellulaire, de clonage des gènes et de mise en œuvre d'anticorps monoclonaux ouvrent certainement la possibilité d'élucider à brève échéance certains des aspects de cette régulation. L'étude des bases moléculaires de l'adaptation des enzymes digestives aux conditions alimentaires promet d'être riche en enseignements sur la régulation de l'expression des gènes chez les eucaryotes et d'aboutir à des applications intéressantes dans le domaine de la nutrition humaine et animale.

Pour des raisons de commodité, les enzymes pancréatiques et intestinales seront traitées séparément. Dans le premier cas, quelques données précises sur les mécanismes d'adaptation enzymatique ont été récemment acquises et seront donc discutées. Nos connaissances sur les hydrolases digestives intestinales sont encore assez restreintes en raison de la plus grande complexité de ces molécules et de leur caractère membranaire. Quelques laboratoires se sont penchés sur certains aspects de leur biosynthèse et sur les possibilités de la moduler.

\section{Facteurs influençant les niveaux des enzymes digestives.}

La biosynthèse des enzymes pancréatiques et intestinales est soumise à de nombreuses modulations par des facteurs génétiques, hormonaux et nutritionnels.

L'existence de formes isoenzymatiques de l'amylase, par exemple, dans une proportion génétiquement établie (Bloor, Meisler et Nielsen, 1981) est une caractéristique de l'espèce. Cependant, l'activation de l'expression des gènes de cette enzyme pourra se traduire par des fluctuations des taux relatifs des ARNm correspondants et, par conséquent, du niveau d'activité amylasique (Owerbach, Nielsen et Rutter, 1981). Des insuffisances en lactase et sucrase-isomaltase, deux disaccharidases de la bordure en brosse intestinale, sont à l'origine de troubles pathologiques chez l'homme (Kretchmer, 1971), mais sont aussi naturelles dans certains groupes ethniques comme les esquimaux (Skovbjerg, Gudmand-Høyer et Fenger, 1980). Pendant l'ontogénèse chez le rat, les niveaux des enzymes pancréatiques sont considérablement augmentés $\left(10^{3}-10^{4}\right.$ fois) au cours des deux derniers jours de la vie fœtale (Rutter et al., 1968). Chez le rat nouveau-né, l' $\alpha$-amylase et la chymotrypsine sont prédominantes, mais leur activité diminue de façon importante 2 à 4 jours après la parturition (Robberecht et al., 1971). De même, l'épithélium intestinal subit de profondes modifications de son ultrastructure et de son équipement enzymatique, tant pendant la période pré-natale que post-natale (Henning, 1981). La lactase et la sucrase-isomaltase, toutes deux ancrées dans la membrane apicale de l'entérocyte, sont considérées comme des marqueurs de différenciation cellulaire. Le niveau de la première enzyme est maximal à la naissance et décroît ensuite alors que la seconde activité n'apparaît nettement qu'au moment du sevrage (Doell et Kretchmer, 1962).

Parmi les hormones ayant une action sécrétagogue sur le pancréas, les peptides intestinaux du type de la cholécystokinine (CCK) modifient aussi à long terme 
la biosynthèse des enzymes pancréatiques. L'injection par voie intraveineuse, en continu, de pancréozymine à des doses physiologiques augmente la biosynthèse des enzymes pancréatiques chez le rat anesthésié (Reggio, Cailla-Deckmyn et Marchis-Mouren, 1971) alors que la caeruléine à plus forte dose augmente spécifiquement la biosynthèse du trypsinogène et diminue de manière importante celle de l'amylase chez le rat éveillé (Schick, Kern et Scheele, 1984 a). Si le glucagon est connu pour inhiber la synthèse protéique pancréatique (Adler, 1977), l'insuline augmente la biosynthèse de l'amylase chez le rat diabétique uniquement. En effet, on sait que le diabète alloxanique du rat entraîne une diminution de la synthèse de cette enzyme et une augmentation de celles du trypsinogène et du chymotrypsinogène et que des injections journalières de l'hormone réversent ces effets (Ben Abdeljlil, Palla et Desnuelle, 1965 ; Palla, Ben Abdeljlil et Desnuelle, 1967). II faut enfin remarquer que l'action de l'hormone, également observée sur des préparations d'acini isolés de rats diabétiques (Korc et al., 1981) ne se produit pas chez l'animal normal.

Jusqu'à présent, seule la stimulation de la lactase par le jeûne a pu être reliée a l'action d'hormones thyroidiennes chez le rat adulte (Raul et al., 1983). On pense que la diminution du taux des hormones T3 et T4 circulantes, consécutive au jeûne (Goodman et al., 1980), est à l'origine de la stimulation de cette enzyme car l'injection préalable de thyroxine empêche l'augmentation de l'activité de la lactase intestinale. II a été récemment montré que les corticostéroïdes sont capables de moduler l'expression de l'aminopeptidase membranaire chez le rat éveillé (Miura et al., 1983).

Avant d'aborder une étude plus détaillée de l'influence des facteurs nutritionnels sur la biosynthèse des enzymes digestives pancréatiques et intestinales, soulignons que la modulation de ces enzymes est soumise aux rythmes nycthéméraux (Girard-Globa et Bourdel, 1973 ; Girard-Globa et Simond-Cote, 1977 ; Ishikawa et al., 1983 ; Saito et al., 1975).

\section{Régulation nutritionnelle des enzymes pancréatiques.}

3.1. Fluctuations des niveaux enzymatiques. - Les protéines pancréatiques sécrétoires sont stockées dans des granules de zymogènes au sein des cellules acineuses avant d'être déversées dans les canalicules pancréatiques pour constituer, avec les produits de sécrétion des cellules ductales, le suc pancréatique. $\mathrm{Ce}$ séjour intracellulaire d'un mélange protéique complexe explique sans doute l'absence de modification qualitative à court terme du suc (Tartakoff et al., 1975 ; Kraehenbuhl, Racine et Jamieson, 1977). A plus long terme, par contre, l'ingestion de quantités importantes de protéines, glucides et lipides entraîne une augmentation des activités enzymatiques impliquées dans l'hydrolyse de ces substrats (Grossman, Greengard et Ivy, 1943 ; Ben Abdeljil et Desnuelle, 1964). Cinq à sept jours sont en effet nécessaires pour que l'adaptation de la biosynthèse des protéines pancréatiques à un nouveau régime soit totale chez le rat (Ben Abdeljlil et Desnuelle, 1964) ou le porc (Corring et Saucier, 1972). Comme l'indique le tableau 1, les modifications des niveaux enzymatiques dans le tissu et le suc pancréatique s'accompagnent d'une modification de la vitesse de biosynthèse des

Reproduction, Nutrition, Développement n 4 B-1985. -5 . 
TABLEAU 1

Modification de la vitesse de biosynthèse des protéines pancréatiques sécrétoires par la composition du régime alimentaire (D'après Reboud, Pasero et Desnuelle, 1964).

\begin{tabular}{|c|c|c|c|c|c|}
\hline \multirow{2}{*}{ Protéine } & \multicolumn{2}{|c|}{$\begin{array}{l}\text { Radioactivité incorporée } \\
\text { pendant } 15 \mathrm{~min}\left(\times 10^{-3}\right)\end{array}$} & \multicolumn{3}{|c|}{ Zymogène/protéines totales } \\
\hline & HP & HG & HP & $H G$ & $\mathrm{HP} / \mathrm{HG}$ \\
\hline Protéines totales & 700 & 555 & - & - & - \\
\hline Chymotrypsinogène & 266 & 88 & 0.38 & 0.16 & 2.5 \\
\hline Trypsinogène-1 & 47 & 29 & 0.07 & 0.05 & 1.4 \\
\hline Trypsinogène-2 & 70 & 58 & 0.10 & 0.10 & 1.0 \\
\hline
\end{tabular}

Des groupes de 10 rats respectivement adaptés à des régimes hyperprotéiques (HP) et hyperglucidiques (HG) reçoivent $4 \mu$ moles de $\left[{ }^{14} \mathrm{C}\right] \mathrm{D}$, L-valine $(5 \mathrm{mCi} / \mathrm{mmole})$ par voie intrapéritonéale.

enzymes (Reboud, Pasero et Desnuelle, 1964). L'étude de la cinétique d'adaptation de la biosynthèse des protéines pancréatiques chez le rat, d'abord adapté à un régime hyperglucidique puis passé sur un régime hyperprotéique et vice-versa, montre que les vitesses relatives de synthèse de l'amylase, du chymotrypsinogène et du trypsinogène commencent à être modifiées dès la deuxième heure après le changement alimentaire (fig. 1). Cette rapidité d'adaptation (Dagorn et Lahaie, 1981), également soulignée par d'autres auteurs (Deschodt-Lanckman et al., 1971) se répercute sur les niveaux enzymatiques de la glande, deux heures plus tard. Après $24 \mathrm{~h}$ d'adaptation aux nouvelles conditions alimentaires, les changements observés dans les vitesses relatives de synthèse et les teneurs enzymatiques sont tout à fait significatives. II convient de souligner ici que le remplacement dans le régime alimentaire de l'amidon par le glucose (Ben Abdeljlil et Desnuelle, 1964 ; Robberecht et al., 1971) et des protéines par des peptides ou des acides aminés

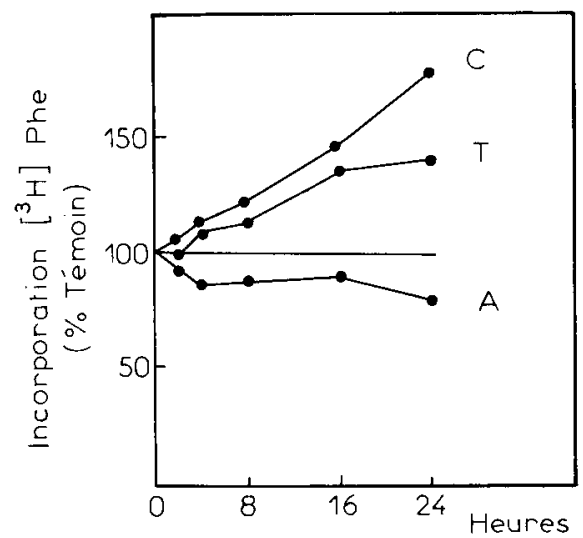

FIG. 1. - Vitesses relatives de synthèse de que/ques protéines sécrétoires chez le rat. Deux groupes de 20 rats sont adaptés à un régime hyperglucidique pendant 15 jours. Après $15 \mathrm{~h}$ de jeûne, l'un est à nouveau alimenté avec le même régime témoin et l'autre, avec un régime hyperprotéique. Dix minutes avant d'être sacrifié, chaque animal reçoit $30 \mu \mathrm{Ci}$ de $\left[{ }^{3} \mathrm{H}\right]$ Phe par voie intrapéritonéale. Les pancréas sont alors homogénéisés, les protéines séparées par électrofocalisation sur gel de polyacrylamide et la radioactivité de chaque bande est déterminée par comptage en scintillation liquide. Les lettres A, C, T correspondent respectivement à l'amylase, au chymotrypsinogène et au trypsinogène ( $d$ 'après Dagorn et Lahaie, 1981). 
(Grossman, Greengard et Ivy, 1943 ; Ben Abdeljil et Desnuelle, 1964) entraîne une stimulation comparable des activités concernées.

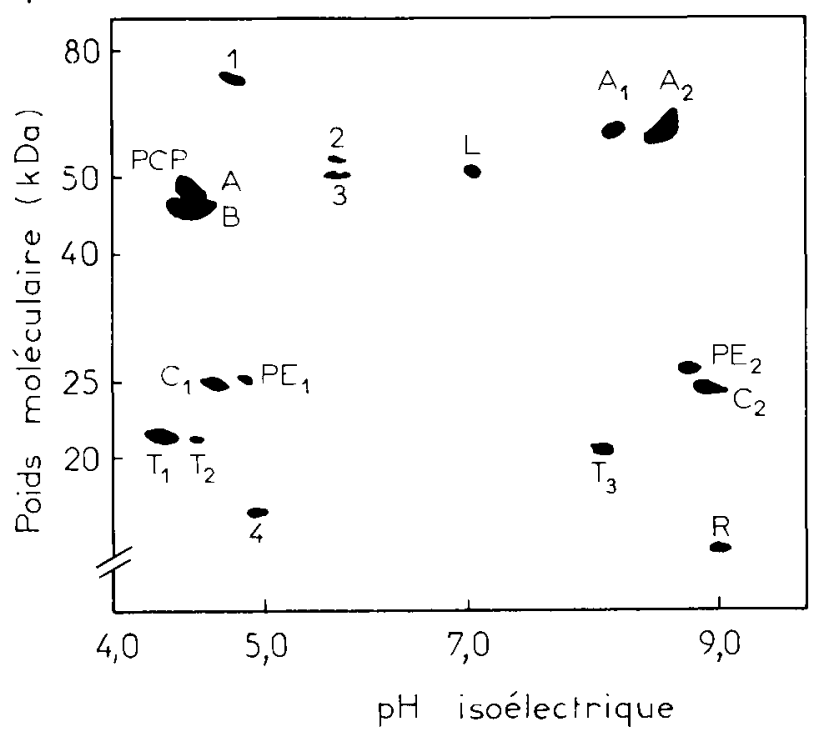

FIG. 2. - Séparation bidimensionnelle des protéines sécrétoires du pancréas de rat Wistar. Les protéines solubles extraites avec un tampon à pH alcalin sont d'abord séparées par électrofocalisation puis par électrophorèse sur gel de polyacrylamide (gradient de 10 à $20 \%$ ) en présence de $0,2 \%$ SDS (dodécyl sulfate de sodium). Le mélange d'ampholines (LKB) comprend : $0.33 \% \mathrm{pH} 3$. $5-10.0,0.33 \% \mathrm{pH} 5.0-8.0,0.5 \% \mathrm{pH} 4.0-6.0$ et $0.4 \% \mathrm{pH}$ 9.0-11.0. Les protéines sont colorées au bleu de Coomassie, révélées en 1ere dimension par des substrats spécifiques. Une vingtaine de protéines sont ainsi identifiées : 2 amylases (A1, A2), 2 chymotrypsinogènes (C1, C2), 2 proélastases (PE1, PE2), 1 procarboxypeptidase A (PCPA), 3 procarboxypeptidases B (PCP B), 1 lipase (L), 1 ribonucléase (R), 3 trypsinogènes (T1, T2, T3). Les protéines 1 à 3 , non encore identifiées correspondent à des glycoprotéines (d'après Scheele, 1975).

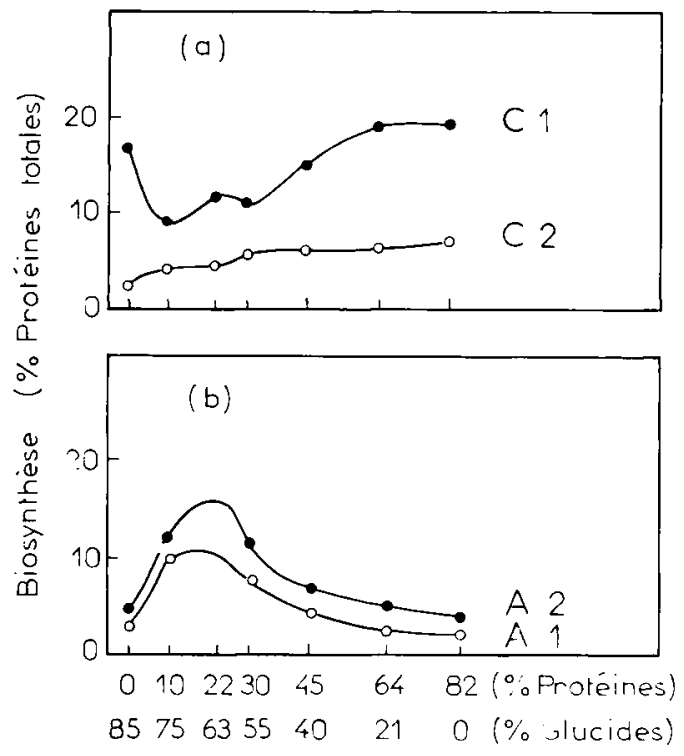

FIG. 3. - Influence de la teneur en protéines et glucides du régime alimentaire sur les vitesses relatives de biosynthèse du chymotrypsinogène (a) et de l'amylase (b) chez le rat. Des préparations de lobules pancréatiques provenant d'animaux adaptés pendant 12 jours aux différentes conditions nutritionnelles sont incubées in vitro pendant $120 \mathrm{~min}$ dans une solution de Krebs-Ringer HEPES à pH 7.4 en présence d'un mélange des 20 acides aminés dont 15 sont marqués au ${ }^{14} \mathrm{C}$. Après séparation des protéines par électrofocalisationélectrophorèse sur gel de polyacrylamide en présence de SDS et comptage de la radiocativité, la biosynthèse relative de chacune des isoenzymes est exprimée en \% de la radioactivité totale incorporée dans l'ensemble des protéines sécrétoires (d'après Schick et al., 1984 b). 
Ainsi que le montre la figure 2, la séparation effective des différentes isoenzymes du pancréas a pu être récemment réalisée par électrofocalisation et électrophorèse en présence de SDS (dodécyl sulfate de sodium) par Scheele (1980). Des gène structuraux multiples semblent être à l'origine de ce polymorphisme. L'étude de la réponse des différentes isoenzymes des protéases pancréatiques à des changements de régime montre que c'est surtout la synthèse des formes acides des isoezymes qui est modulée (Schick et al., 1984 b). La biosynthèse du chymotrypsinogène anionique $\mathrm{C} 1$ et de son homologue cationique $\mathrm{C} 2$ est respectivement augmentée de 1,6 fois et 1,1 fois quand les animaux reçoivent le régime hyperprotéique ( $82 \%$ caséine) par rapport au régime normal. Dans les même conditions expérimentales, les biosynthèses relatives des amylases, toutes deux cationiques, varient parallèlement (fig. 3 ).

3.2 Mécanismes de l'adaptation enzymatique dans le pancréas. - L'augmentation du niveau des enzymes pancréatiques en réponse à la consommation de régimes riches en protéines, glucides ou lipides, résulte d'une modification spécifique de leur vitesse de biosynthèse (Reboud, Pasero et Desnuelle, 1964). La régulation nutrionnelle de la teneur en ARN messager de la cellule acineuse pancréatique peut provenir d'un effet au cours de la transcription de I'ADN en ARN hétérogène nucléaire, de la maturation de ce dernier en ARNm fonctionnel ou encore de la modification de la stabilité du messager proprement dit. Une modulation de la traduction du messager en protéine correspondra aussi à une modification de l'expression du gène eucaryote considéré. L'expression génétique est donc soumise à de multiples contrôles.

Des études d'adaptation nutrionnelle utilisant l'actinomycine $D$, un inhibiteur de la synthèse des $A R N m$, semblent indiquer que la vitesse de synthèse du trypsinogène-1 chez le rat est indépendante de la synthèse de l'ARN correspondant au cours de l'adaptation de cette protéine à un régime contenant de l'inhibiteur trypsique de soja (Dijkhof et Poort, 1978). A l'aide d'actinomycine D, administrée à la dose de $2 \mathrm{mg} / \mathrm{Kg}$ à des rats recevant un régime normal ou hyperprotéique, nous avons pu montrer que seule la stabilité des ARNm du trypsinogène-1 et de la proélastase-1 est modifiée (tabl. 2) et que celle des ARNm des autres protéines

TABLEAU 2

Influence des conditions nutritionnelles sur les demi-vies des ARN messagers pancréatiques (D'après Wicker et Puigserver, 1985 a).

\begin{tabular}{lcc} 
ARN messager & \multicolumn{2}{c}{ Demi-Vies (h) } \\
\cline { 2 - 3 } & Régime standard & Régime hyperprotéique \\
\hline Amylase & $5.5-5.6$ & $5.1-5.3$ \\
Lipase & $3.3-4.0$ & $3.7-4.0$ \\
Procarboxypeptidase & $3.2-3.4$ & $3.8-4.0$ \\
Protéases à sérine & $3.8-3.9$ & $3.7-3.9$ \\
Proélastase & $6.5-6.7$ & $3.0-3.6$ \\
Trypsinogène-1 & $3.0-3.5$ & $5.0-5.9$ \\
Chymotrypsinogènes-1 et 2 & $3.6-3.8$ & $3.5-4.2$ \\
Trypsinogène 3 & $3.2-4.1$ & $3.2-3.2$ \\
\hline
\end{tabular}


pancréatiques ne l'est pas (Wicker et Puigserver, 1985 a). Ce résultat suggère donc que la régulation nutrionnelle de la biosynthèse de certaines enzymes pancréatiques est au moins en partie, traductionnelle. En fait, un contrôle supplémentaire transcriptionnel des gènes de l'amylase et des protéases à sérine a pu être également démontré en déterminant la quantité d'ARN biologiquement actif (Wicker, Scheele et Puigserver, 1983 ; Wicker, Puigserver et Scheele, 1984) ou en quantifiant les ARNm à l'aide de sondes d'ADN complémentaires (Giorgi et al., 1984).

Les techniques classiques de préparation des ARN messagers à partir de pancréas de rat sont assez délicates à mettre en œuvre en raison du taux élevé de ribonucléase dans ce tissu. On peut souligner aussi la faible intensité de la réponse adaptative aux conditions alimentaires par opposition aux inductions de la biosynthèse protéique chez les bactéries où les niveaux de production des protéi-

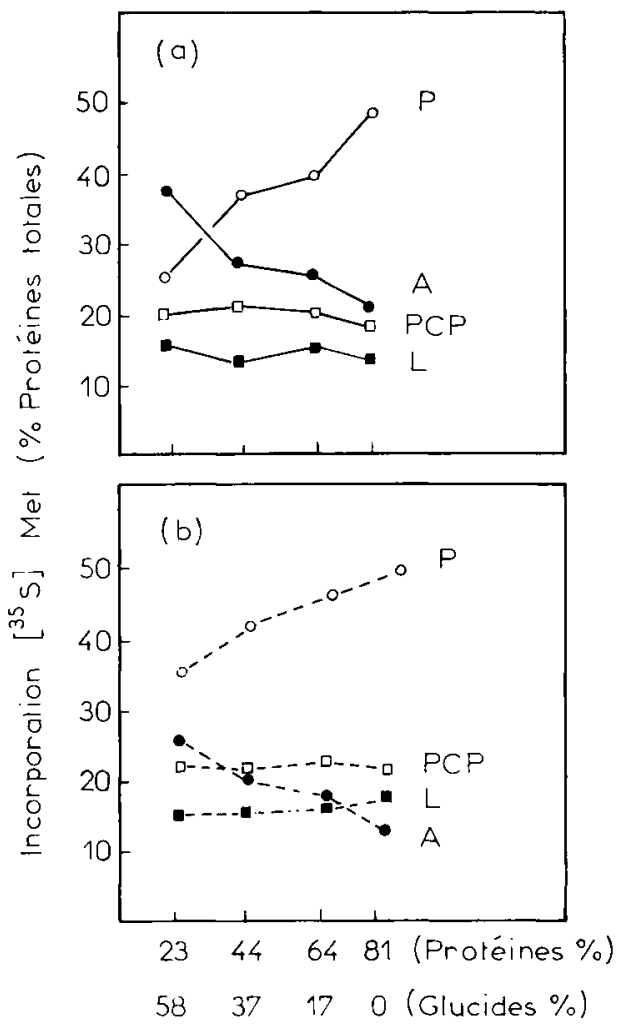

FIG. 4. - Comparaison de la biosynthèse relative de quelques protéines sécrétoires par des lobules pancréatiques isolés (a) ou par traduction in vitro des ARN messagers correspondants (b). Les lobules pancréatiques ou les ARN totaux obtenus à partir d'animaux adaptés pendant 9 jours aux différents régimes alimentaires sont incubés en présence de $\left[{ }^{35} \mathrm{~S}\right]$ méthionine. Les protéines résultantes sont séparées par électrophorèse monodimensionnelle sur gel de polyacrylamide en présence de SDS. Après prélèvement des bandes de gel correspondant à chaque protéine et dissolution par $\mathrm{H}_{2} \mathrm{O}_{2}$ à $50^{\circ} \mathrm{C}$, la radioactivité est déterminée et exprimée en \% de la radioactivité totale incorporée dans l'ensemble des protéines. Chaque point représente la moyenne de 4 animaux. La synthèse des protéines en milieu acellulaire est réalisée dans le système de lysats de réticulocytes de lapins (d'après Wicker, Puigserver et Scheele, 1984). 
nes varient généralement de plus de cent fois. Malgré ces difficultés, il nous a été possible d'établir une bonne corrélation entre les produits de traduction in vitro des ARNm de l'amylase, de la famille des protéases à sérine, des procarboxypeptidases $A$ et $B$ et de la lipase avec les protéines biosynthétisées dans les lobules pancréatiques isolés des mêmes pancréas (fig. 4). Quelles que soient les conditions nutritionnelles utilisées, un contrôle transcriptionnel des gènes de l'amylase et des protéases à sérine est observé.

Enfin, nous avons pu montrer que la caeruléine, un analogue structural de la cholécystokinine, module la biosynthèse des enzymes pancréatiques en exerçant un contrôle au niveau de la traduction des ARNm (Wicker et al., 1985 b). On sait que la caeruléine est un puissant sécrétagogue qui, injecté par voie intraveineuse en continu, est capable de stimuler la synthèse du chymotrypsinogène et du trypsinogène et de diminuer celle de l'amylase dans le pancréas de rat (Schick, Kern et Scheele, 1984 a). Compte tenu de nos observations, il est donc assez peu probable que la cholécystokinine joue un rôle prépondérant comme intermédiaire hormonal de la régulation nutritionnelle de la biosynthèse des enzymes pancréatiques.

\section{Adaptation des hydrolases intestinales au régime alimentaire.}

4.1. Cas des hydrolases membranaires. - La muqueuse intestinale est organisée en villosités à la base desquelles se trouvent des cryptes dont l'épithélium est constitué de cellules indifférenciées capables de se diviser très activement. La migration des cellules le long de l'axe villositaire permet un renouvellement rapide de l'épithélium intestinal (environ 2 jours chez le rat). A ces transformations morphologiques est notamment associée l'apparition des enzymes digestives membranaires qui sont toutes des glycoprotéines. L'aminopeptidase et la dipeptidylpeptidase IV sont des exopeptidases, la phosphatase alcaline intervient dans la déphosphorylation des phosphoprotéines et phospholipides alimentaires, quant au complexe sucrase-isomaltase, il représente l'une des enzymes responsables de l'activité disaccharidasique intestinale.

Liées à la maturation des entérocytes le long de l'axe villositaire, les activités et quantités immunoréactives des hydrolases intestinales présentent un gradient croissant des cryptes au sommet des villosités (Raul et al., 1977 ; Boyle, Celano et Koldovský, 1980). Des variations sont observées selon les enzymes considérées. Ainsi, alors que le niveau de la sucrase est maximal dès la mi-hauteur de la villosité, la lactase et l'aminopeptidase sont plus représentées dans la partie apicale des villosités.

Tout ce qui précède montre que l'organisation morphologique et fonctionnelle de la muqueuse intestinale est complexe et que ses hydrolases constitutives, des glycoprotéines membranaires de haut poids moléculaire, sont difficiles à purifier à l'état natif, ce qui a longtemps retardé la réalisation d'études précises sur leur adaptation nutritionnelle. Cependant, l'amélioration des méthodes biochimiques de dosage des activités enzymatiques et le développement récent de méthodes immunoélectrophorétiques (Skovbjerg, Norén et Sjöström, 1978) et radioimmunologiques (Miura et al., 1983) aboutit déjà à des études fort intéressantes dans ce domaine. 
La régulation nutritionnelle des peptidases membranaires a été l'objet d'un nombre assez restreint de travaux comparés à ceux entrepris sur les disaccharidases. Sans jeûne préliminaire, une augmentation d'environ deux fois de l'activité aminopeptidasique est observée au niveau de l'intestin grêle chez des rats nourris pendant 5 jours avec des régimes contenant environ 55 à $90 \%$ de protéines (Nicholson, Mc Carthy et Kim, 1974). Une étude segmentale de la réponse peptidasique mesurée à l'aide du substrat phénylalanylglycine (Mc Carthy, Nicholson et Kim, 1980) montre que cette stimulation se produit préférentiellement au niveau de l'iléon (fig. 5).

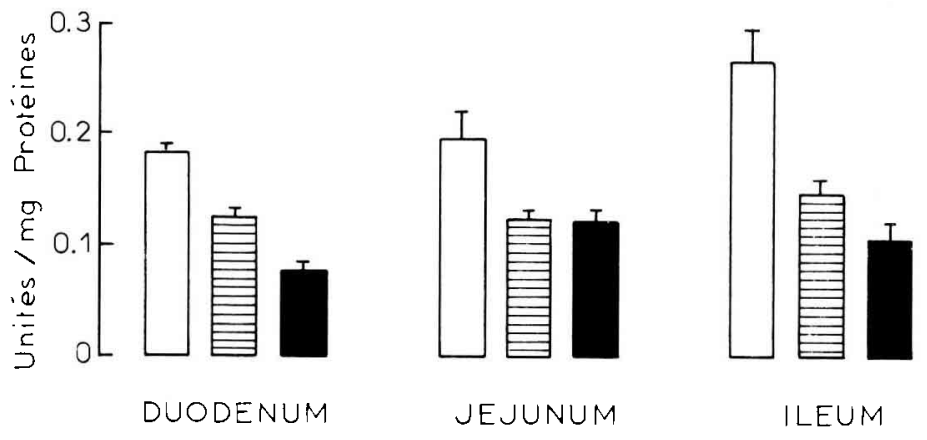

FIG. 5. - Adaptation au régime alimentaire de l'aminopeptidase intestinale au niveau du duodénum, du jejunum et de l'iléum chez le rat. Après 7 jours d'adaptation à des régimes contenant $65 \%$ de protéines (口), $72 \%$ de glucides ( $\square$ ) et $72 \%$ de lipides (目), les muqueuses intestinales sont récoltées et l'activité enzymatique est estimée par hydrolyse de la L-phénylalanylglycine. Six rats mâles Wistar sont utilisés par groupe et les différences observées sont significatives pour un niveau de probabilité de $95 \%(\mathrm{p}<0,05)$ (d'après Mc Carthy, Nicholson et Kim, 1980).

Le passage d'un régime normal à un régime déficient en glucide, hyperprotéique ou hyperlipidique, entraîne une diminution des activités disaccharidasiques de l'intestin. Inversement, chez des animaux à jeun ou carencés en glucides, l'ingestion d'un régime riche en glucides augmente l'activité de ces enzymes chez le rat ou l'homme (Rosenweig et Herman, 1969 et 1970). Les niveaux de la sucraseisomaltase et de la lactase (Raul et al., 1982 ; Yamada, Bustamante et Koldovský, 1981) sont respectivement augmentés de deux et trois fois chez le rat après une quinzaine d'heures et deux jours d'alimentation hyperglucidique. En ce qui concerne la lactase, on sait que le jeûne produit une stimulation comparable à celle d'un régime hyperglucidique (Yamada et al., 1983; Ulshen et Grand, 1979). II semble cependant que l'activité lactasique intestinale soit rapidement lenviron 12 h) modulable par un régime hyperglucidique (Goda et al., 1984). Enfin, soulignons le fait que la localisation de la réponse enzymatique le long de l'axe villositaire a été étudiée. Après une période de jeûne, l'ingestion de sucrose augmente l'activité de la saccharase principalement dans les cellules immatures des cryptes et la partie basse des villosités (Raul et al., 1982 ; Yamada et al., 1983 ; Ulshen et Grand, 1979). L'adaptation gagnerait les cellules du sommet de la villosité grâce à la migration entérocytaire.

La plus grande partie des études décrites ci-dessus a été réalisée en déterminant des activités enzymatiques à partir d'homogénats de muqueuse intestinale et, 
quelquefois seulement, des bordures en brosse purifiées. En utilisant une méthode immunoélectrophorétique, il a été possible récemment de quantifier la réponse des hydrolases membranaires à différentes conditions nutritionnelles en les précipitant sélectivement par leurs anticorps spécifiques (Gaucher, 1984). II est

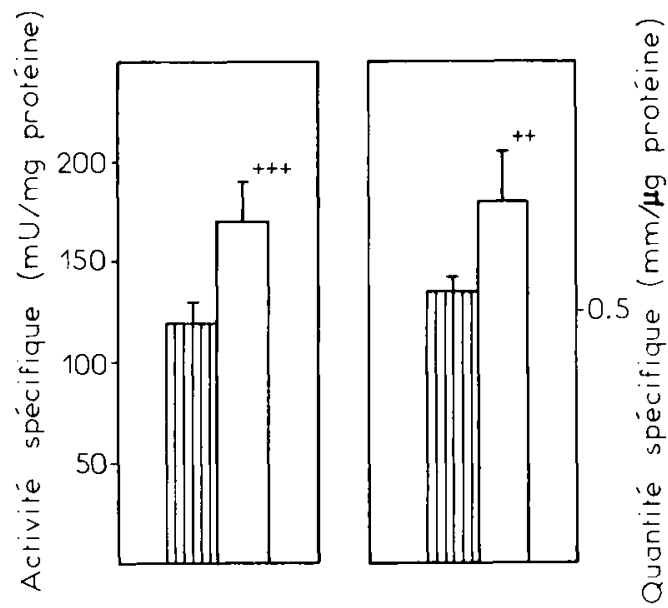

FIG. 6. - Activité et quantité spécifiques de l'aminopeptidase de la bordure en brosse intestinale au cours d'une alimentation hyperprotéique. Un groupe de 8 rats mâles Wistar reçoit pendant 4 jours une alimentation protéique normale $(10 \%)$ tandis que le second groupe est nourri pendant deux jours avec l'aliment à $10 \%$ de protéines et les deux jours suivants avec l'aliment hyperprotéique $(65 \%)$. Après récolte des muqueuses intestinales et homogénéisation en présence de $2 \%$ de détergent (émulphogène), une centrifugation à $105000 \mathrm{~g}$ pendant $50 \mathrm{~min}$ à $4^{\circ} \mathrm{C}$ permet d'obtenir des surnageants sur lesquels l'aminopeptidase est dosée par méthode enzymatique et par voie immunoélectrophorétique. Le dosage enzymatique est réalisé sur le substrat synthétique leucinep-nitroalilide. La quantification immunoélectrophorétique de cette hydrolase fait appel a la méthode des immunoélectrophorèses en " roquettes " (une seule dimension), la migration s'effectuant dans un gel d'agarose contenant les anticorps précipitants. La hauteur des pics d'immunoprécipités $(\mathrm{mm})$ est proportionnelle à la quantité d'enzyme. Les différentes observées sont significatives avec $p<0,02\left(^{* *}\right)$ et $p<0,001(* * *)$ (d'après Gaucher, 1984).

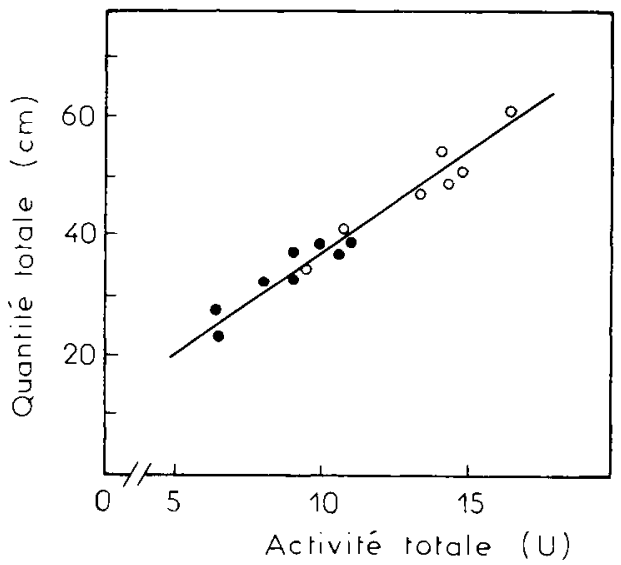

FIG. 7. - Droite de régression linéaire quantité-activité de l'aminopeptidase intestinale. Les données expérimentales sont celles de la Figure 6 avec $p<0.001$ et un coefficient de corrélation $r=0.97$ pour les animaux témoins $(\bullet)$ et ceux recevant le régime hyperprotéique (0) (d'après Gaucher, 1984). 
d'ailleurs possible d'utiliser un mélange d'anticorps dirigés contre des bordures en brosses purifiées et d'identifier ensuite les hydrolases gråce à leur réaction avec un substrat chromogénique spécifique. Comme il est indiqué sur la figure 6 , la variation de la hauteur du pic d'immonoprécipité de l'aminopeptidase intestinale, quand on passe d'une régime normal à un régime hyperprotéique, est comparable à celle des activités enzymatiques. Une très bonne corrélation entre quantité immunoréactive d'enzyme et capacité hydrolytique est obtenue (fig. 7). On peut donc en conclure que l'adaptation des enzymes intestinales au régime alimentaire résulte d'un effet sur leur vitesse de biosynthèse.

4.2. Bases moléculaires de l'adaptation enzymatique intestinale. - Des études moléculaires aussi avancées que celles réalisées dans le cas des enzymes pancréatiques sont encore inexistantes, en raison principalement de la masse moléculaire plus élevée des protéines concernées, de leur très faible importance pondérale et de la difficulté à maîtriser les expériences de traduction en milieu acellulaire des ARN messagers intestinaux.

En quantifiant les hydrolases intestinales au cours de l'adaptation, comme déjà indiqué (Gaucher, 1984), il a été possible de montrer qu'une modulation s'exerce sur les niveaux des protéines biosynthétisées. L'utilisation de méthodes différentes a conduit d'autres auteurs à la même conclusion (Nsi-Emvo et Raul, 1984 ; Cézard et al., 1983). Avec des inhibiteurs spécifiques de la transcription et de la tracduction, comme l'actinomycine $D$ et la cycloheximide, la caractérisation partielle et indirecte des étapes affectées au cours de la réponse adaptative a pu être obtenue. Ainsi, l'effet inhibiteur de l'actinomycine D s'exercerait sur l'ensemble de l'axe villositaire (Raul et al., 1982), ce qui semble donc indiquer, dans le cas des disaccharidases tout au moins, que la régulation nutritionnelle par le saccharose s'exerce au niveau transcriptionnel. II semble en effet que dans des conditions expérimentales comparables, la biosynthèse des peptidases et de la phosphatase de la bordure en brosse intestinale ne soit pas effectée par cet antibiotique. D'autres types de régulations s'exerceraient donc à un niveau différent dans ce cas, peut-être par le biais de la stabilité des ARN messagers correspondants. La cycloheximide, quant à elle, pourrait inhiber l'ensemble des activités enzymatiques.

La modification des activités polymérasiques associées à la chromatine a été récemment étudiée (Raul et Von Der Decken, 1983 et 1984) car elles sont impliquées dans les processus de transcription. D'après ces études, il semblerait que la réalimentation par du saccharose pur, de rats à jeun depuis $48 \mathrm{~h}$, provoque une stimulation des activités polymérasiques impliquées dans la transcription des ARN préribosomaux (I) et messagers (II). Le jeûne diminue fortement les activités non dissociées des polymérases I et III, mais une réalimentation par du saccharose pendant $15 \mathrm{~h}$ est capable de rétablir à $60 \%$ l'activité globale de départ. La polymérase II, insensible au jeûne, est stimulée à environ $30 \%$ par l'ingestion de saccharose. De plus, la quantité libre de polymérase II non engagée dans un processus transcriptionnel est réduite au cours du jeûne et augmente beaucoup après ingestion de saccharose, sans toutefois dépasser le niveau basal. Cette substance exercerait donc un effet plus spécifique sur la transcription des ARN messagers 
(polymérase II) comparés aux ARN ribosomaux qui sont affectés par d'autres conditions alimentaires (polymérase 1). La stimulation transcriptionnelle pourrait se produire à la fois dans les entérocytes matures et les cellules indifférenciées des villosités (Raul, communication personnelle).

Si une régulation transcriptionnelle des disaccharidases semble assez plausible, le mécanisme précis de ce type de contrôle n'est pas pour autant élucidé. Pour les autres hydrolases, notre ignorance des bases moléculaires des phénomènes d'adaptation nutritionnelle est encore plus grande. II est toutefois raisonnable de penser que des progrès décisifs seront rapidement faits dans ce domaine grâce à la mise en œuvre des techniques modernes de la biologie moléculaire et de l'immonologie. L'équipement enzymatique de la cellule intestinale est aujourd'hui assez bien défini et la masse moléculaire élevée des protéines actives n'apparaît certainement plus comme un problème insurmontable.

\section{Conclusion.}

L'ensemble des travaux réalisés à ce jour sur les phénomènes de régulation nutritionnelle des hydrolases pancréatiques et intestinales est considérable. II n'en reste pas moins vrai que les informations obtenues sont surtout descriptives et que les mécanismes régissant ces phénomènes d'adaptation enzymatique sont probablement très complexes et encore presque totalement inconnus. S'agissant d'enzymes d'origine cellulaire différente, mais de spécificités complémentaires, il est difficile de prévoir si les mécanismes sous-jacents de la modulation au niveau pancréatique et intestinal sont identiques ou différents. II est cependant assez probable que la régulation de la biosynthèse d'une enzyme digestive ne fasse pas intervenir un contrôle à un niveau unique mais résulte d'une combinaison d'actions, plus ou moins prononcées suivant les cas, au niveau transcriptionnel ou traductionnel et à celui de la stabilité des ARN messagers ou de leurs produits de traduction. Quel que soit le mécanisme, l'aliment ingéré agira indirectement sur la cellule cible. Ainsi, les produits de la digestion des protéines et des glucides sont connus pour exercer les mêmes effets que les macromolécules de départ (Ben Abdeljil et Desnuelle, 1964 ; Robberecht et al., 1971 ; Corring 1977). Enfin, la production d'effecteurs au niveau intestinal, consécutive à l'ingestion d'un aliment ou à sa digestion, pourrait constituer une étape supplémentaire dans la modulation du niveau des enzymes digestives (Simoes-Nunes, 1982 ; Desseaux, SimoesNunes et Marchis-Mouren, 1984).

Journees Ingestion, Digestion, Absorption de l'Association française de Nutrition, Versailles, 24-25 octobre 1984.

\section{Références}

ADLER G., 1977. Effect of glucagon on the secretory process in the rat exocrine pancreas. Cell Tiss. Res., 182, 193-204.

BEN ABDELJLIL A., DESNUELLE P., 1964. Sur l'adaptation des enzymes exocrines du pancréas à la composition du régime. Biochim. biophys. Acta, 81, 136-149.

BEN ABDELJLIL A., PALLA J. C., DESNUELLE P., 1965. Effect of insulin on pancreatic amylase and chymotrypsinogen. Biochem. biophys. Res. Commun., 18, 71-75. 
BLOOR J. H., MEISLER M. H., NIELSEN J. T., 1981. Genetic determination of amylase synthesis in the mouse. J. biol. Chem., 256, 373-377.

BOYLE J. T., CELANO P., KOLDOVSKY O., 1980. Demonstration of a difference in expression of maximal lactase and sucrase activity along the villus in the adult rat jejunum. GastroenterologY, 79, 503-507.

CEZARD J. P., BROYART J. P., CUISINIER-GLEIZE S. P., MATHIEU H., 1983. Sucrase-isomaltase regulation by dietary sucrose in the rat. Gastroenterology, 84, 18-25.

CORRING, T., 1977. Possible role of hydrolysis products of the dietary components in the mechanisms of the exocrine pancreatic adaptation to the diet. WId. Rev. Nutr. Diet., 27, 132-144.

CORRING T., SAUCIER R., 1972. Sécrétion pancréatique sur porc fistulé. Adaptation à la teneur en protéines du régime. Ann. Biol. anim. Bioch. Biophys., 12, 223-241.

DAGORN J. C., LAHAIE R. G., 1981. Dietary regulation of pancreatic protein synthesis. I. Rapid and specific modulation of enzyme synthesis by changes in dietary composition. Biochim. biophys. Acta., 654, 111-118.

DESCHODT-LANCKMAN M., ROBBERECHT P., CAMUS J., CHRISTOPHE J., 1971. Short-term adaptation of pancreatic hydrolases to nutritional and physiological stimuli in adult rats. Biochimie, 53, 789-796.

DESSEAUX, V., SIMOES-NUNES, C., MARCHIS-MOUREN, G., 1984. Adaptation du pancréas exocrine du porc au régime alimentaire : mise en évidence d'une fraction peptidique active obtenue à partir de l'intestin grêle proximal. Résultats préliminaires. Reprod. Nutr. Dévelop., 24, 781.

DIJKHOF J., POORT C., 1978. Changes in rat pancreatic protein synthesis after a single feeding with diets containing raw or heated soybeans. J. Nutr., 108, 1222-1228.

DOELL R. G., KRETCHMER N., 1962. Studies of small intestine during development. I. Distribution and activity of $\beta$-galactosidase. Biochim. biophys. Acta, 62, 353-362.

GAUCHER C., 1984. Régulation nutritionnelle des enzymes de la bordure en brosse intestinale chez le rat en croissance. Th. Doc.-Ing. Univ. Aix-Marseille II.

GIORGI D., BERNARD J. P., LAPOINTE R., DAGORN J. C., 1984. Regulation of amylase messenger RNA concentration in rat pancreas by food content. EMBO J., 3, 1521-1524.

GIRARD-GLOBA A., BOURDEL G., 1973. Phisiological regulation of the circadian rhythm in hepatic tyrosine transmaminase : schedule of protein ingestion as a determinant factor. J. Nutr., 103, 251-256.

GIRARD-GLOBA A., SIMOND-COTE E., 1977. Nutritional and circadian variation in lipase activity and colipase saturation in rat pancreas. Ann. Biol. anim. Bioch. Biophys., 17, 539-542.

GODA T., BUSTAMANTE S., THORNBURG W., KOLDOVSKY O., 1984. Dietary-induced increase in lactase activity and immunoreactive lactase in adult rat jejunum. Biochem. J., 221, 261-263.

GOODMAN M. N., LARSEN P. R., KAPLAN M. M., AOKI T. T., YOUNG V. R., RUDERMAN N. B., 1980. Starvation in the rat. II. Effect of age and obesity on protein sparing and fuel metabolism. Am. J. Physiol., 239, E227-286.

GROSSMAN M. I., GREENGARD H., IVY A. C., 1943. The effect of dietary composition of pancreatic enzymes. Am. J. Physiol., 138, 676-682.

HENNING S. J., 1981. Post natal development : coordination of feeding, digestion and metabolism. Am. J. Physiol., 241, G199-214.

ISHIKAWA Y., SUZUKI M., ONO A., NAKABOU Y., HAGIHIRA H., 1983. Memory of the rhythmic change of duodenal alkaline phosphatase in rats. J. Nutr. Sci. Vitaminol., 29, 85-90.

KORC M., IWAMOTO Y., SANKARAN H., WILLIAMS J. A., GOLDFINE I. D., 1981. Insulin action in pancreactic acini from streptozotocin-treated rats. I. Stimulation of protein synthesis. Am. J. Physiol., 240, G56-62.

KRAEHENBUHL J. P., RACINE L., JAMIESON J. C., 1977. Immunocytochemical localization of secretory proteins in bovine pancreatic exocrine cells. J. Cell Biol., 72, 406-423.

KRETCHMER N., 1971. Memorial lecture : lactose and lactase - a historical perspective. Gastroentorology, 61, 805-813.

Mc CARTHY D. M., NICHOLSON J. A., KIM Y. S., 1980. Intestinal enzyme adaptation to normal diets of different composition. Am. J. Physiol., 239, G445-451.

MIURA S., MORITA A., ERICKSON R. H., KIM Y.S., 1983. Content and turnover of rat intestinal microvillus membrane aminopeptidase. Effect of methylprednisolone. Gastroenterology, 85. $1340-1349$. 
NICHOLSON J. A., Mc CARTHY D. M., KIM Y. S., 1974. The responses of rat intestinal brush border and cytosol peptide hydrolase activities to variation in dietary protein content. J. clin. Invest., 54, 890-898.

NSI-EMVO E., RAUL F., 1984. Stimulation of lactase synthesis induced by starvation in the jejunum of adult rat. Enzyme, 31, 45-49.

OWERBACH D., NIELSEN J. T., RUTTER W. J., 1981. On the mechanism of variation of pancreatic amylase levels in mouse strains. J. biol. Chem., 256, 6502-6506.

PALLA J. C., BEN ABDELJLIL A., DESNUELLE P., 1967. Comparative study of the control of amylase biosynthesis in rat pancreas and parotid glands. Biochim. biophys. Acta., 136, 563-565.

RAUL F., NORIEGA R., NSI-EMVO E., DOFFOEL M., GRENIER J. F., 1983. Lactase activity is under hormonal control in the intestine of adult rat. Gut, 24, 648-652.

RAUL F., POUSSE A., GRENIER J.F., HAFFEN K., 1982. La stimulation de l'activité de la saccharase par le saccharose est-elle sous la dépendance d'une synthèse d'ARN dans l'intestin de rat adulte ? Gastroent. clin. biol., 6, 424-429.

RAUL F., SIMON P. M., KEDINGER M., HAFFEN K., 1977. Intestinal enzyme activities in isolated villus and crypt cells during postnatal development of the rat. Cell Tiss. Res., 176, 167-178.

RAUL F., VON DER DECKEN A., 1983. Modulation of RNA polymerase activities in the intestine of adult rats by dietary sucrose. J. Nutr., 113, 2134-2140.

RAUL F., VON DER DECKEN A., 1984. Dietary sugar promotes gene activation in intestinal cell chromatin of adult rats. Experientia, 40, 364-365.

REBOUD J. P., PASERO L., DESNUELLE P., 1964. On chymotrypsinogen and trypsinogen biosynthesis by pancreas of rats fed on a starch-rich diet or a casein-rich diet. Biochem. biophys. Res. Commun., 17, 347-351.

REGGIO H., CAILLA-DECKMYN H., MARCHIS-MOUREN G., 1971. Effect of pancreozymin on rat pancreatic enzyme biosynthesis. J. Cell Biol., 50, 333-343.

ROBBERECHT P., DESCHODT-LANCKMAN M., CAMUS J., BRUYLANDS J., CHRISTOPHE J., 1971. Rat pancreatic hydrolases from birth to weaning and dietary adaptation after weaning. Am. J. Physiol., 221, 376-381.

ROSENWEIG N. S., HERMAN R. H., 1969. Time response of jejunal sucrase and maltase activity to a high sucrose diet in normal man. Gastroenterology, 56, 500-505.

ROSENWEIG N. S., HERMAN R. H., 1970. Dose response of jejunal sucrase and maltase activities to isocaloric high and low carbohydrate diets in man. Am. J. clin. Nutr., 23, 1373-1377.

RUTTER W. J., KEMP J. D., BRADSHAW W. S., CLARK W. R., RONZIO R. A., SANDERS T. G., 1968. Regulation of specific protein synthesis in cytodifferentiation. J. Cell Physiol., 72, Suppl., 1-18.

SAITO M., MURAKAMI E., NISHIDA T., FUJISAWA Y., SUDA M., 1975. Circadian rhytms in digestive enzymes in the small intestine of rats. I. Patterns of the rhytms in various regions of the small intestine. J. Biochem., 78, 475-480.

SCHEELE G., 1975. Two dimensional gel analysis of soluble proteins : characterization of guinea pig exocrine pancreatic proteins. J. Biol. Chem., 250, 5375-5385.

SCHEELE G. A., 1980. Biosynthesis, segregation and secretion of exportable proteins by the exocrine pancreas. Am. J. Physiol., 238, G467-477.

SCHICK J., KERN H., SCHEELE G., 1984 a. Hormonal stimulation in the exocrine pancreas results in coordinate and anticoordinate regulation of protein synthesis. J. Cell Biol., 99, 1569-1574.

SCHICK J., VERSPOHL R., KERN H., SCHEELE G., 1984 b. Two distinct adaptative responses in the synthesis of exorcrine pancreatic enzymes to inverse changes in protein and carbohydrate in the diet. Am. J. Physiol. 247, G611-616.

SIMOES-NUNES, C., 1982. Lack of pancreatic enzyme adaptation to dietary carbohydrate and lipid after proximal small intestine by-pass in the pig. Digestion, 25, 108.

SKOVBJERG H., GUDMAND-H $\varnothing$ YER E., FENGER H. J., 1980. Immunoelectrophoretic studies on human small intestinal brush border protein-amount of lactase protein in adult-type hypolactasia. Gut, 21, 360-364.

SKOVBJERG H., NORÉN O., SJOSSTROMM H., 1978. Immunoelectrophoretic studies on human small intestinal brush border proteins. Scand. J. clin. Lab. Invest. 38, 723-729.

TARTAKOFF A. M., JAMIESON J. D., SCHEELE G. A., PALADE G. E., 1975. Studies on the pancreas of the guinea pig. Parallel processing and discharge of exocrine protein. J. biol. Chem., 250, 2671-2677. 
ULSHEN M. H., GRAND R. J., 1979. Site of substrate stimulation of jejunal sucrase in the rat. J. clin. Invest., 64, 1097-1102.

WICKER C., PUIGSERVER A., 1985 a. Selective dietary modulation in the stability of some pancreatic mRNAs coding for serine protease zymogens on the rat. Soumis a Biochem. $J$.

WICKER C., PUIGSERVER A., RAUSCH U., SCHEELE G., KERN H., 1985 b. Multiple-Level caerulein control of the gene expression of secretory proteins in the rat pancreas. Eur. J. Biochem. (sous presse).

WICKER C., PUIGSERVER A., SCHEELE G., 1984. Dietary regulation of levels of active mRNA coding for amylase and serine protease zymogens in the rat pancreas. Eur $J$. Biochem., 139, 381387.

WICKER C., SCHEELE G., PUIGSERVER A., 1983. Adaptation au régime alimentaire du niveau des ARNm codant pour l'amylase et les protéases à sérine pancréatiques chez le rat. $C$. R. Acad. Sci. Paris Ser. D Sci. Nat., 297, 281-284.

YAMADA K., BUSTAMANTE S., KOLDOVSKY O., 1981. Dietary-induced rapid increase of rat jejunal sucrase and lactase activity in all regions of the villus. FEBS Lett., 129, 89-92.

YAMADA K., GODA T., BUSTAMANTE S., KOLDOVSKY O., 1983. Different effect of starvation on activity of sucrase and lactase in rat jejunoileum. Am. J. Physiol., 244, G449-455. 